\title{
Effectiveness of Pelvic Proprioceptive Neuromuscular Facilitation Technique on Facilitation of Trunk Movement in Hemiparetic Stroke Patients
}

\author{
Dildip Khanal ${ }^{1}$, R. M. Singaravelan ${ }^{2}$, and Subhash M. Khatri ${ }^{3}$ \\ ${ }^{\text {I}}$ Postgraduate student, College of Physiotherapy, PIMS, Loni- 413 736, India) \\ ${ }^{2}$ (Associate professor, College of Physiotherapy, PIMS, Loni- 413 736, India) \\ ${ }^{3}$ (Professor and Principal, College of Physiotherapy, PIMS, Loni- 413 736, India)
}

\begin{abstract}
Background: As the pelvic motion comes from trunk muscles, a specific use of pelvic pattern not only exercises the pelvis for mobility and stability but also facilitate trunk motion and stability.Objective: To determine the effect of Pelvic Proprioceptive Neuromuscular Facilitation(PNF) technique on facilitation of trunk movement in hemiparetic stroke patients.Interventions: Thirty hemiparetic stroke patients were randomly divided into two groups. The experimental group received pelvic PNF while the control group received conventional physiotherapy in the form of truncal exercises for 30 minutes. Along with these both group received regular physiotherapy in the form of tonal management and range of motion exercises for the affected limbs for 30 minutes. Intervention was given once in a day for five days/week for four weeks.Outcome Measures: Trunk Impairment Scale (TIS), Trunk Lateral Flexion Range of Motion (TLF ROM), and Tinetti Test (TT). Results: Post-intervention, both the groups shows improvement on trunk performance, range of motion, balance and gait but the experimental group shows more improvement than control group [changes in mean score between group comparison for TIS 3.4 (1.8); TLF ROM for affected and non-affected side 0.734 \& 3.666 (0.267 \& 2.533); Balance 3.534 (2.266) and Gait 2.2 (1)]. The level of significance was set at $p<0.05$ and highly significance at $p<0.01$.
\end{abstract}

Key words:Inclinometer, Pelvic Proprioceptive Neuromuscular Facilitation, Stroke, Tinetti Test, Trunk Impairment Scale, Trunk Lateral Flexion Range of Motion

\section{Introduction}

Stroke is a common neurological disorder, representing a major cause of disability. It isconsidered as a significant health problem, which needs an unremitting and wide-ranging rehabilitation [1].Stroke is also known as "cerebral vascular accident", "brain attack" or "apoplexy" [2,3]. According to WHO stroke is defined as "acute onset of neurological dysfunction due to abnormality in cerebral circulation with resultant signs and symptoms that corresponds to involvement of focal area of brain lasting more than 24 hours" [4]. Approximately 700,000 individuals in United States are affected by it each year.About 500,000 are new strokes and 200,000 are recurrent strokes [5]. According to W.H.O (16 November 2011) the incidence of stroke in India was 130/100,000 individuals every year [6].The Indian Council of Medical Research estimates that among the non-communicable disease, stroke contributes for $41 \%$ of deaths and $72 \%$ of disability adjusted life years $[7,8]$.

Following the stroke trunk function is also impaired along with impairment of limb function. Trunk muscle strength when measured by using isokinetic dynamometer and hand-held dynamometershowed that trunk muscle strength was impaired multidirectionally while performing activities such as flexion, extension, lateral flexion and rotation [9-11]. As a result, trunk asymmetry appears bearing great percentage of body weight on an unaffected limb than on the affected limb [12].Concerning the range of motion (ROM) of the trunk in stroke patients, Mohrstated that "If there is not full range in all trunk movements (flexion, extension, lateral flexion and rotation), it will be more difficult to gain full control of the trunk. Any lack of ROM in trunk will lead to decreased function" [13]. A study done by Verheyden et al.showed anaffiliation between trunk performance and balance, gait \& functional ability after stroke [14]. In addition, the trunk performance is also considered as a key prophet for the functional outcome after stroke [15-17].

Pelvis is a part of trunk that supports extremity motions. Hence, the pelvic motion comes from trunk muscles. The range of motion in the pelvic patterns depends on the amount of motion in the lower spine. Biomechanically it is impossible to move the pelvis without motion in the spine as it is connected with the spine. Specific pelvic patterns of Proprioceptive Neuromuscular Facilitation (PNF), which are mentioned in the literature not only exercise the pelvis motion and stability but also facilitate trunk motion and stability. In addition, these patterns help to improve functional trunk activities and treat the upper trunk \& cervical areas indirectly through irradiation [18]. 
Various authors have investigated the effects of pelvic PNF technique on gait pattern and have found improvement in cadence, stride length and gait duration; but there is hardly any study that has investigated its effectiveness in trunk movements that is essential for normal symmetry of body, balance and in functional activities. Hence, this study was aimed to find out the effects of pelvic PNF technique on facilitation of trunk movement in hemiparetic stroke patients.

\section{Methods}

2.1 Participants: This study is a randomized control study, which includes baseline, pre-interventional evaluation, intervention, post-interventional evaluation. Thirty hemiparetic subacute stroke participants who participated in the intervention were recruited from the Neuro physiotherapy department, Pravara Institute of Medical Sciences, Loni (Bk)-413 736, Taluka Rahata, District Ahmednagar, Maharashtra State, India. Study received an ethical clearance from the Institutional Ethical Committee. The participants were screened to ensure that they met the following inclusion criteria: Both male and female participants with stroke involving Middle Cerebral Artery (MCA) territory on Magnetic Resonance Imaging findings or Computerized Tomography Scan, the age group between 45 to 60 years, first ever unilateral stroke, the stroke duration within 6 months, no previous PNF treatment given to the pelvic region since 6 weeks, able to ambulate 10 meters with or without walking aids. The exclusion criteria were participants who had aphasia, not medically stable, uncontrolled hypertension, spinal deformity, pelvis fracture less than 6 months, impaired cognitive function, maximum score on trunk impairment scale and trunk lateral flexion range of motion at the start of the study. The informed consent was obtained from the participants before they were enrolled into the intervention [19,20]. The participants included in the study were then randomly assigned either to the experimental group (pelvic PNF) or to the control group (conventional physiotherapy).

2.2 Outcomes Measure:The Trunk Impairment Scale (TIS) - The TIS assesses static sitting balance, dynamic sitting balance, and trunk coordination on a scale ranging from 0 to 23 points. The maximum score on the subscales of the TIS are 7, 10, and 6 points respectively. A higher score indicates a better trunk performance [21]. Trunk Lateral Flexion Range of Motion (TLF ROM)was measured by using inclinometer [22,23], and Tinetti Test (TT)- The TT was used to document the participants balance and gait performance. The balance subscale consists of 9 balance tasks. The score ranges from 0 to 16 points. The gait subscale evaluates 7 gait maneuvers. The score ranges from 0 to 12 points. Consequently, the total Tinetti Test score ranges between 0 to 28 points. The higher score indicating a better performance [24]. All these outcomes measure were used to evaluate the trunk performance, trunk lateral flexion ROM, balance and gait respectively before the intervention and after 4 weeks of intervention.

2.3 Intervention: The participants of experimental group received Pelvic PNFfor 30 minutes, once in a day, 5 days per week for 4 weeks $[20,25]$. The elements of PNF such as positioning, manual contact, stretch, resistance and verbal commands were incorporated into the treatment. The pelvic patterns used were anterior elevation and posterior depression. The techniques used in this study were rhythmic initiation, slow reversal and agonistic reversal. The sequence used was rhythmic initiation, slow reversal andagonistic reversal. Each sequence was performed for total of 10 minutes including the two minutes of rest interval in each sequence. The participant's position used in this study was side lying position, which allows free motion of the pelvis \& easy reinforcement of trunk and lower extremity activities [18].

All the participants in the control group received conventional physiotherapy in the form of truncal exercises, which consisted task-specific exercises of the upper and lower part of the trunk. The exercises were performedboth in the supine and in sitting positions. It was performed for total of 30 minutes including 6 minutes of rest period in between as per the patients requirement,once in a day, 5 days per week for 4 weeks. It was performed on the plinth.The exercises in supine were as follows: bridging; unilateral bridging;in crook lying position - lifting pelvis and placing left and right of midline, lifting shoulder girdle asymmetrically and symmetrically from the plinth; and the trunk rotation initiated from upper and lower part of the trunk. The exercises in sitting were as follows: flexion and extension of the trunk (the participant were asked to flexed and extends the trunk without moving the trunk forward or backward);trunk lateral flexion initiated from the shoulder and pelvic girdle (from the shoulder girdle means -participant touches the plinth with one elbow and returns to the starting position, from the pelvic girdle means-participant lifts one side of the pelvis and returns to the starting position); rotation from the upper part of trunk (from the upper part of the trunk means- participant moves each shoulder forward and backward); rotation from the lower part of the trunk (from the lower part of the trunk means- participant moves each knee forward and backward); and shuffling the pelvis forward, backward and one side to other on a plinth.The truncal exercises were performed with the assistance initially if required and progressed to a state of no assistance. The therapist based on the participant's performance determined the numbers of repetitions of the exercise [25-27]. 
Along with the above exercises, both the group received regular physiotherapy treatment in the form of tonal management and range of motion exercises for affected side limbs for 30 minutes.

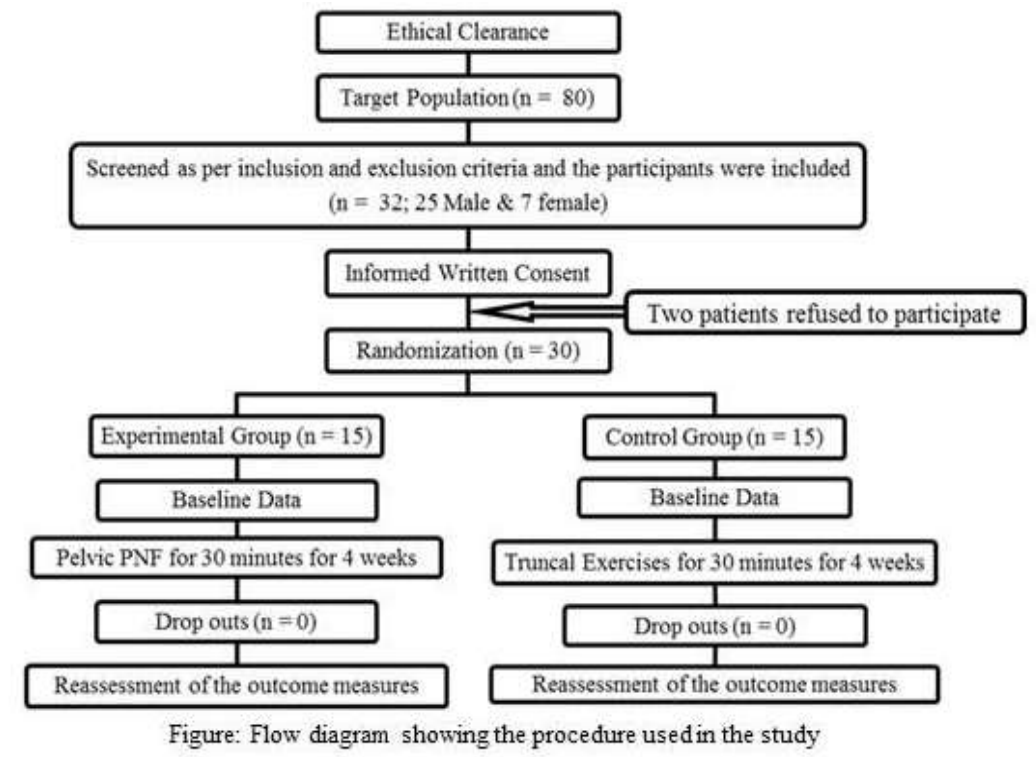

III. Data Analysis and Results

Data were analyzed using Graph Pad InStat software - Trial version 3.10. The data were entered into an excel spreadsheet, tabulated and subjected to statistical analysis. Various statistical measures were used for this study which includes mean, standard deviation (SD) and test of significance such as paired ' $\mathrm{t}$ ' \& unpaired ' $t$ ' test.

\subsection{Demographics and clinical characteristics of stroke:}

The comparison between groups for baseline demographic and clinical characteristics of stroke such as age, sex, duration of stroke, affected side, type of stroke (Hemorrhagic/Ischemic), and use of walking aids were analyzed by descriptive statistics. The baseline demographic and clinical characteristics between the groups were evaluated by unpaired ' $t$ ' test. Pre and post interventional outcome measures score within a group were analyzed by Paired't' test. Unpaired't' test was used to compare the difference in scores between the two groups i.e. the Experimental group and the Control group. For this purpose, the primary investigator collected data and the comparison of scores was made between the pre intervention and after 4 weeks of post intervention. The results were concluded to be statistically significant with $\mathrm{p}<0.05$ and highly significant with $\mathrm{p}<0.01$. (Table 3.1)

Table 3.1: Demographic and clinical characteristics of stroke

\begin{tabular}{|c|l|c|c|c|}
\hline $\begin{array}{c}\text { Sr. } \\
\text { No. }\end{array}$ & \multicolumn{1}{|c|}{ Demographic Profile } & Experimental Group & Control Group & $\begin{array}{c}\text { P } \\
\text { Value }\end{array}$ \\
\hline 1. & Age (years) & $49.133 \pm 4.779^{\mathrm{a}}$ & $50.066 \pm 6.123^{\mathrm{a}}$ & $0.3226^{\mathrm{c}}$ \\
\hline 2. & Sex (Male/Female) & $11(73.33 \%) / 4(26.66 \%)^{\mathrm{b}}$ & $12(80 \%) / 3(20 \%)^{\mathrm{b}}$ & - \\
\hline 3. & Duration of stroke (days) & $96.533 \pm 34.477^{\mathrm{a}}$ & $92.266 \pm 33.892^{\mathrm{a}}$ & $0.7351^{\mathrm{c}}$ \\
\hline 4. & Affected Side (Right/Left) & $8(53.33 \%) / 7(46.66 \%)^{\mathrm{b}}$ & $9(60 \%) / 6(40 \%)^{\mathrm{b}}$ & - \\
\hline 5. & Type of stroke (Haemorragic/ Ischemic) & $1(6.66 \%) / 14(93.33 \%)^{\mathrm{b}}$ & $2(13.33 \%) / 13(86.66 \%)^{\mathrm{b}}$ & - \\
\hline 6. & Use of walking aids & 3 & 5 & - \\
\hline
\end{tabular}

Where: ${ }^{a}$. Values expressed as Mean \pm Standard Deviation

b. Values expressed in percentage

'. Analyzed by Student's Unpaired 't' test

\subsection{Trunk Impairment Scale (TIS):}

The trunk performance was measured with the help of TIS score. The difference in the pre and postinterventional mean score for the experimental group was 3.4. The difference in the pre and post-interventional mean score for the control group was 1.8. (Table 3.2) 
Table 3.2: Comparison of TIS score pre \& post-interventional between experimental and control group

\begin{tabular}{|c|c|c|c|}
\hline $\begin{array}{l}\text { Trunk Impairment Scale } \\
\text { (TIS) }\end{array}$ & $\begin{array}{l}\text { Experimental } \\
\text { Group }\end{array}$ & ControlGroup & $\begin{array}{c}\text { Comparison } \\
\text { between }\end{array}$ \\
\hline Pre-interventional Score & $6.467 \pm 2.295^{\mathrm{a}}$ & $6.600 \pm 2.028^{\mathrm{a}}$ & Experimental Group \\
\hline Post-interventional Score & $9.867 \pm 2.503^{\mathrm{a}}$ & $8.400 \pm 2.028^{\mathrm{a}}$ & \&Control Group \\
\hline 't' value & $14.466^{\mathrm{d}}$ & $6.081^{\mathrm{d}}$ & $1.763^{\mathrm{c}}$ \\
\hline $\mathrm{P}$ value & $<0.01^{\mathrm{d}}$ & $<0.01^{\mathrm{d}}$ & $<0.05^{\mathrm{c}}$ \\
\hline Results & Highly Significant & $\begin{array}{c}\text { Highly } \\
\text { Significant }\end{array}$ & Significant \\
\hline
\end{tabular}

Where: ${ }^{\mathrm{d}}$. Analyzed by Student's Paired't' test

\subsubsection{Subscales of Trunk Impairment Scale:}

The subscale of TIS includes Static Sitting Balance (SSB), Dynamic Sitting Balance (DSB) and Trunk Coordination (TC). The difference in the pre and post-interventional mean score of the experimental group for SSB and DDB was 3.363 and 3.5 respectively. The difference in the pre and post-interventional mean score of the control group for SSB and DSB was 1.9 and 1.6 respectively. (Table 3.2.1)

Table 3.2.1: Comparison of SSB \& DSB score subscales of TIS pre and post-interventional between experimental and control group

\begin{tabular}{|l|c|c|c|c|}
\hline \multirow{2}{*}{ Subscales of TIS } & \multicolumn{2}{|c|}{ Static Sitting Balance (0-7) } & \multicolumn{2}{c|}{ Dynamic Sitting Balance (0-10) } \\
\cline { 2 - 5 } & Experimental Group & Control Group & ExperimentalGroup & ControlGroup \\
\hline Pre-interventional Score & $5.364 \pm 1.120^{\mathrm{a}}$ & $5.500 \pm 1.269^{\mathrm{a}}$ & $2.500 \pm 1.915^{\mathrm{a}}$ & $1.800 \pm 1.304^{\mathrm{a}}$ \\
\hline Post-interventional Score & $8.727 \pm 1.555^{\mathrm{a}}$ & $7.400 \pm 1.430^{\mathrm{a}}$ & $6.000 \pm 1.826^{\mathrm{a}}$ & $3.400 \pm 1.517^{\mathrm{a}}$ \\
\hline 't' value & $10.864^{\mathrm{d}}$ & $6.042^{\mathrm{d}}$ & $12.124^{\mathrm{d}}$ & $0.0389^{\mathrm{d}}$ \\
\hline P value & $<0.01^{\mathrm{d}}$ & $<0.01^{\mathrm{d}}$ & $<0.01^{\mathrm{d}}$ & $<0.05^{\mathrm{d}}$ \\
\hline Results & Highly Significant & Highly Significant $^{\mathrm{d}}$ & Highly Significant $^{\text {Significant }}$ \\
\hline
\end{tabular}

\subsection{Trunk Lateral Flexion Range of Motion (TLF ROM):}

TLF ROM was measured with double inclinometer.

3.3.1 TLF ROM for the Experimental Group:The difference in the pre and post-interventional mean score of TLF ROM in the affected side and non-affected side was 0.734 and 3.666 respectively. (Table 3.3.1)

Table 3.3.1: TLF ROM of affected and non-affected side of experimental group

\begin{tabular}{|l|c|c|}
\hline TLF ROM for Experimental Group & Affected Side ROM & Non-Affected Side ROM \\
\hline Pre-interventional & $8.933 \pm 3.283^{\mathrm{a}}$ & $7.067 \pm 2.915^{\mathrm{a}}$ \\
\hline Post-interventional & $9.667 \pm 2.895^{\mathrm{a}}$ & $10.733 \pm 2.987^{\mathrm{a}}$ \\
\hline ' $\mathrm{t}$ ' value & $2.442^{\mathrm{d}}$ & $12.084^{\mathrm{d}}$ \\
\hline P value & $<0.05^{\mathrm{d}}$ & $<0.01^{\mathrm{d}}$ \\
\hline Results & Significant & Highly Significant \\
\hline
\end{tabular}

3.3.2 TLF ROM for the Control Group:The difference in the pre and post-interventional mean score of TLF $\mathrm{ROM}$ in the affected side and non-affected side was 0.267 and 2.533 respectively. (Table 3.3.2)

Table 3.3.2: TLF ROM of affected and non-affected side of control group

\begin{tabular}{|l|c|c|}
\hline \multicolumn{1}{|c|}{ TLF ROM for Control Group } & Affected Side ROM & Non Affected Side ROM \\
\hline Pre-interventional & $9.133 \pm 2.850^{\mathrm{a}}$ & $6.000 \pm 2.878^{\mathrm{a}}$ \\
\hline Post-interventional & $9.400 \pm 2.667^{\mathrm{a}}$ & $8.533 \pm 2.264^{\mathrm{a}}$ \\
\hline ' $\mathrm{t}$ ' value & $2.256^{\mathrm{d}}$ & $8.718^{\mathrm{d}}$ \\
\hline P value & $<0.05^{\mathrm{d}}$ & $<0.01^{\mathrm{d}}$ \\
\hline Results & Significant & Highly Significant \\
\hline
\end{tabular}

Table 3.3.3: Comparison of TLF ROM between the experimental and the control group

\begin{tabular}{|l|c|c|}
\hline $\begin{array}{c}\text { Comparison of TLF ROM between } \\
\text { Experimental Group \& Control Group }\end{array}$ & Affected Side & Non-Affected Side \\
\hline ' $\mathrm{t}$ ' value & $0.2624^{\mathrm{c}}$ & $2.273^{\mathrm{c}}$ \\
\hline $\mathrm{P}$ value & $>0.05^{\mathrm{c}}$ & $<0.05^{\mathrm{c}}$ \\
\hline Results & Not Significant & Significant \\
\hline
\end{tabular}




\subsection{Tinetti Test (TT):}

The balance and gait was evaluated with the help of Tinetti Test. The difference in the pre and postinterventional means score for the experimental group was 5.734. The difference in the pre and postinterventional mean score for the control group was 3.4. (Table 3.4)

Table 3.4: Comparison of TT score pre \& post-interventional between experimental and control group

\begin{tabular}{|l|c|c|c|}
\hline \multicolumn{1}{|c|}{ Tinetti Test (TT) } & $\begin{array}{c}\text { Experimental } \\
\text { Group }\end{array}$ & Control Group & $\begin{array}{c}\text { ComparisonbetweenExperimental } \\
\text { Group\&Control Group }\end{array}$ \\
\cline { 1 - 2 } $\begin{array}{l}\text { Pre-interventional } \\
\text { Score }\end{array}$ & $9.933 \pm 2.120^{\mathrm{a}}$ & $10.267 \pm 2.492^{\mathrm{a}}$ & \\
\cline { 1 - 2 } $\begin{array}{l}\text { Post-interventional } \\
\text { Score }\end{array}$ & $15.667 \pm 2.440^{\mathrm{a}}$ & $13.600 \pm 2.354^{\mathrm{a}}$ & \\
\hline 't' value & $14.938^{\mathrm{d}}$ & $15.811^{\mathrm{d}}$ & $2.361^{\mathrm{c}}$ \\
\hline P value & $<0.01^{\mathrm{d}}$ & $<0.01^{\mathrm{d}}$ & $<0.05^{\mathrm{c}}$ \\
\hline Results & Highly Significant & $\begin{array}{c}\text { Highly } \\
\text { Significant }\end{array}$ & Significant \\
\hline
\end{tabular}

\subsection{Discussion:}

\section{IIII. Discussion and Conclusion}

Most of the studies in stroke rehabilitation are concerned withthe management of the upper or lower extremity dysfunction. In contrast with limb rehabilitation, trunk restoration is a rather neglected area of stroke rehabilitation research. Hence, this study aimed to evaluate the effects of Pelvic Proprioceptive Neuromuscular Facilitation (PNF) technique on facilitation of trunk movement in hemiparetic stroke patients. Both the group result shows improvement in terms of trunk performance, trunk lateral flexion range of motion, balance and gait when assessed by Trunk Impairment Scale, Inclinometer and Tinetti Test. However, the overall improvement in the experimental group who received pelvic PNF was greater than control group who received truncal exercises in the form of conventional physiotherapy.

4.1.1 Experimental Group:The result of this study shows that there was improvement in all the outcome measures in the experimental group when evaluated by Trunk Impairment Scale, Trunk Lateral Flexion Range of Motion and Tinetti Test. This could be due to the effects of Proprioceptive Neuromuscular Facilitation, which was applied to the pelvic region.

There was 1.888 times more improvement in trunk impairment scale in the experimental group compared to control group suggesting better trunk muscle activity. The probable mechanism by which PNF could have worked is by facilitating the neuromuscular mechanism, thus by stimulating the proprioceptors. Kabat reported that a greater motor response can be attained when employing facilitating techniques in addition to resistance. Facilitation resulted from a number of factors such as application of stretch, use of particular movement patterns and use of maximal resistance in order to induce irradiation.Gellhorn and Loofbourroe showed that when a muscle contraction is resisted, that muscle's response to cortical stimulation increases. The use of particular movement patterns also causes changes in spinal and supraspinal level $[5,18]$. All these facilitatory techniques might help to facilitate trunk motion and stability, treat upper trunk and cervical areas indirectly through irradiation thus enhancing the motor control and motor learning thereby improving performance of participants in the experimental group on TIS. A study done by Deletis, Dimitrijevic, Sherwood and Kaisai et al. explained in detail about neuromuscular mechanism. They stated that in PNF position, sensory inputs from the periphery leads to stronger excitation of the cortical areas, leading to variations in the thresholds of a number of motoneurons, which was reflected in the motor evoked potentials [28].This was further supported by a study of Benecke, Meyer, Gohman and Conrad that the amount of sensory input coming from the periphery was greater in PNF position than in normal position, which induces changes in the excitability of the pyramidal tract, the final motor pathways [29].In addition, a study both electrical by Merton \& Morton and transcranial magnetic stimulation by Barker et al. produce complex descending corticospinal volleys which usually contain a direct component via corticospinal neurons and an indirect trans-synaptic component [3032].Even the treatment methods that allegedly are based on neurophysiological principles, however, do not have a fully comprehensive and experimentally proven neurophysiological basis. Neurophysiological approaches, however, focus on upgrading of the lost motor capacities. In that sense, Knott and Voss referred to "hidden potentials" of the patients for the recovery [33].

Over a period of 20-treatment session, the mean score on the SSB improved from 5.364 to 8.727.This shows that there was 1.77 times more improvement in SSB in the experimental group compared to control group. The SSB shows noteworthy result because the mean score on the pretreatment was already 5.364 out of a maximum of 7 points. For dynamic sitting balance, there was improvement of mean score from 2.500 to 6.000 . This shows an improvement of 2.178 times more in the experimental group compared to the control group. On 
the other hand, the participants in this group were not able to score in the trunk coordination that evaluates the coordination of shoulder girdle and pelvic girdle.It is said that the subscales of the trunk impairment scale contain a hierarchy. In a sample of 15 hemiparetic stroke participants, static sitting balance appeared to be easier than dynamic sitting balance and dynamic sitting balance in its turn easier than trunk coordination [34]. This hierarchy possibly explained why the participants in this group demonstrated a beneficial effect for static sitting balance and dynamic sitting balancebut not for trunk coordination. On the other hand, pretreatment scores for static sitting balance were already at a sub-maximal level. In addition, 10 hours of treatment for four weeks of pelvic PNF technique might not have been enough to result an overflow of irradiation to the upper part of trunk to cause a marked change in more obscure coordination items of trunk impairment scale.

In comparison to the control group, there was $2.749 \& 1.447$ times more improvement in affected side and non-affected side TLF ROM respectively in the experimental group. PNF increases the ROM by increasing the length of muscle and the neuromuscular efficiency. The physiological mechanism for increasing the ROM may be due to autogenic inhibition, reciprocal inhibition, and stress relaxation [35]. The techniques which were used in this study i.e. rhythmic initiation, slow reversal and agonistic reversal might help to normalized the tone of affected side trunk muscles, lengthening the contracted structures, relax the hypertonic muscles, help in initiating the movements, strengthening the weak muscles and help in improving the control of the pelvis. All these effects might directly or indirectly aid in improving the trunk lateral flexion range of motion. This was in accordance with the Ruth Dickstein, Shraga Hocherman, and Thomas Pillar, who have done study in stroke rehabilitation using three exercise therapy approaches where they found pattern of muscle tone improvement in the PNF treatment group [36].In addition, in the study that was conducted by Grace McNelis, Chris Pappas, and Tim Josten et al. in gait analysis and PNF, they reported normalization of pelvic motion [37].

There was 1.720 times more improvement in Tinetti test in the experimental group compared to the control group, suggesting carry-over effect of pelvic PNF in improving balance and gait. The PNF approach to treatment uses the principle that control of motion proceeds from proximal to distal body regions. Facilitation of trunk control, therefore, is used to influence the extremities. If this treatment pattern is valid, gaining control of and strengthening, "normal" pelvic motions should improve lower extremity function and balance [3840].Tyson SF in 1999 and Messier S, Bourbonnais D, Desrosiers J et al.studied the trunk kinematics during walking in stroke patients. They reported that there is unstable and asymmetrical pelvic movements during walking and these movements are executed by the upper trunk with very little lower trunk (pelvic movement) after strokethus affecting the balance and gait [41,42]. The result of the present study found improvement in trunk performance in terms of static sitting balance and dynamic sitting balance that positively affects the gait and balance. The similar results found by Trueblood et al. In their study, PNF based resisted anterior elevation and posterior depression of pelvic movements for lower trunk muscles resulted in an improvement in walking in early phase stroke participants [19]. However, the improvement in the entire outcome measures in this study could be due to natural recovery also, as we have recruited the subacute stroke participants.

4.1.2 Control Group:The result of this study shows that there was improvement in all the outcomes measure in the control group when evaluated by Trunk Impairment Scale, Trunk Lateral Flexion Range of Motion and Tinetti Test. This could be due to the effects of truncal exercises, which was given in the form of conventional physiotherapy to the control group.

The improvement in the trunk performance, which was evaluated by trunk impairment scale, could be due to the task-specific practice of truncal exercises aiming to improve trunk performance that may lead to plasticity of the sensorimotor regions of the central nervous system [43]. A study done by John Chae et al. suggests that active repetitive training of the hemiparetic limb shapes subsequent functional reorganization in the adjacent intact cortex and the undamaged motor cortex plays an important role in motor recovery $[44,45]$. According to Classen et al. focal transcranial magnetic stimulation and functional magnetic resonance imaging have shown that task-specific training yields long-lasting cortical reorganization specific to the corresponding areas being used [46]. Karni et al. using functional MRI, and Classen et al. using Transcranial Magnetic Stimulation, both reported a slowly evolving, long-term, experience-dependent reorganization of the adult primary motor cortex after daily practice of task-specific motor activities [46-48].In addition, Van Praag also suggested that repetitive practice increases production of neurotransmitter, such as acetylcholine and serotonin, increases synthesis of neurotroponin-3 that may lead to the proliferation and greater survival of hippocampal progenitor cells, which may be important for new learning and memory [43]. These results are in partial accordance with Geert Verheyden, Luc Vereeck, and Steven Truijen et al. who have reported analogous improvement in trunk performance in stroke participants when given additional 10 hours of truncal exercises and were evaluated by trunk impairment scale [25].

There was also observable improvement in the subscales of trunk impairment scale, which could be explained by the hierarchy in its subscales possibly. Even the pretreatment score for static sitting balance were already at a sub-maximal level for control group. In addition, 10 hours of training might not have been enough 
to result noticeable improvement for more difficult coordination items of TIS. Wim Saeys, Luc Vereeck and Steven Truijen et al explained this higher level of truncal function in their study by giving 16 hours of additional truncal exercises instead of 10 hours to stroke participants. They found significant improvement in dynamic sitting balance and trunk coordination. Furthermore, their study showed that truncal function improved postural control during standing balance and mobility indicating the importance of truncal exercises in the rehabilitation of stroke patients [26].The improvement in the trunk lateral flexion ROM in the control group could be due to the effect of regular or intensive training as the participants were asked to bend their trunk laterally to touch the exercise table with elbow \& return to the initial position. In addition, exercises that were implemented for the control group was focused on coordination, selective muscle strengthening and selective movements of the trunk. Yue and Cole et al. suggested that the gain in strength in stroke patient that increase motor recovery may derive from a variety of mechanism, as practice of motor skills may increase sensorimotor representation and derives from cortical and subcortical levels onto the spinal motor pools, leading to greater recruitment or firing rates of this pool [43].

The improvement in the Tinetti test of the control group shows that the effect of truncal exercises transferred to enhanced balance and ambulation. Trunk is considered as a central key point of the body and it is said that the control of movements proceeds from proximal to distal body regions. Thus, the proximal permanence of the trunk is a requirement for distal limb movement. In addition, truncal stability is acrucial component for balance and coordinated extremity used in daily purposeful activities and the performance in the higher-level motor tasks. Motor control literature also suggest that if an improved level of proximal trunk gains were attained, a better distal limb control might be expected during balance and functional mobility. A cross sectional study by Verheyden et al. also favors the results of this study. In their study, there was a positive association found between the trunk performance, balance and gait after sub-acute stroke [14].The improvement in trunk performance in this study may further aid in improving the balance and gait. In addition, the improvement seen in this study group could be due to natural recovery too, as the participants in this study were in sub-acute stroke stage where the potential chance of recovery lies.

\subsection{Conclusion:}

This four weeks study result showed greater improvement in Pelvic Proprioceptive Neuromuscular Facilitation group in comparison with conventional physiotherapy group in terms of trunk performance, trunk lateral flexion range of motion, balance and gait. Therefore, these results concluded that pelvic Proprioceptive Neuromuscular Facilitation technique is effective on facilitating trunk movement in hemiparetic stroke patients.

\subsection{Implications for practice:}

Pelvic PNF technique can be used along with the regular physiotherapy programme to improve the trunk mobility and stability in order to enhance the balance and the mobility in hemiparetic stroke patients.

\subsection{Limitations of the present study:}

There are certain limitations that warrant caution when generalizing the result of this study. This study included only a small number of participants, only analyzed the results between pre and post treatment values. No follow-up assessment was done to find out the carried over effect, all the participants of our study were recruited from a single geographical location,and though this study showed better trunk muscle activity, it could be studied using surface electromyography (sEMG) for accurate trunk muscle activity in order to isolate the trunk muscle function.

\subsection{Suggestions for future research:}

With multicentre trials, long-term follow-up and evaluating its effects, stroke involving different territory other than MCA

Acknowledgment:I would like to thank Keerthi Rao, Chandra Iyer, Shrikant Sant, Bhushan Vichare and all the participants of this study in helping me to complete the study successfully.

\section{Funding:Nil}

Conflict of interest:None declared

\section{References}

[1] Dally S, and Ruff RL, Electrically induced recovery of gait components for older patients with chronic stroke, Am $J$ Phys Med Rehabil. 79, 2000, 349-60.

[2] Thompson JE, The evolution of surgery for the treatment and prevention of stroke. The Willis Lecture, Stroke 27(8), 1996, 142734. 
[3] Kopito, and Jeff, A Stroke in Time. 6(9), 2001, MERGINET.Com. Available from http://www.webasx.com/articles/strokeintime.html

[4] World Health Organization, Cerebrovascular Disorders Geneva: World Health Organization. 1978 Available from whqlibdoc.who.int/offset/WHO_OFFSET_43.pdf

[5] Susan B O Sullivan and Thomas J Schmitz, Physical Rehabilitation (Fifth edition. New Delhi: Jaypee Brothers Publication; 2007).

[6] Brain stroke third biggest killer in India, health.indiatimes.com/articleshow /1148565.cms

[7] D. Nagaraja, G. Gururaj, N. Girish, Samhita Panda, A.K. Roy, G.R.K. Sarma, R. Srinivasa, Feasibility study of stroke surveillance: Data from Bangalore, India, Indian J Med Res130, 2009, 396-403.

[8] Indian Council for Medical Research,Stroke: Assessment of the burden of Non-communicable diseases: Final project report, New Delhi. Indian Council of Medical Research 2004; 18-22.

[9] Bohannon RW, Lateral trunk flexion strength: impairment, measurement reliability and implications following unilateral brain lesion, Int J Rehabil Res.15, 1992, 249-251.

[10] Tanaka S, Hachisuka K, Ogata H, Trunk rotatory muscle performance in post-stroke hemiplegic patients, Am J Phys Med Rehabil.76, 1997, 366-369.

[11] Tanaka S, Hachisuka K, Ogata H, Muscle strength of trunk flexion-extension in post-stroke hemiplegic patients, Am J Phys Med Rehabil.77, 1998, 288-290.

[12] Dickstein R, Sheffi S, Ben Haim Z, Shabtai E, Markovici E, Activation of flexor and extensor trunk muscles in hemiparesis, Am J Phys Med Rehabil.79, 2000, 228-234.

[13] Mohr JD, Management of the trunk in adult hemiplegia: the Bobath concept, Top Neurol 1(1), 1990, 1-12.

[14] Verheyden G, Vereeck L, Truijen S, et al., Trunk performance after stroke and the relationship with balance, gait and functional ability, Clin Rehabil.20, 2006, 451-458.

[15] Franchignoni FP, Tesio L, Ricupero C, Martino MT, Trunk control test as an early predictor of stroke rehabilitation outcome, Stroke. 28, 1997, 1382-1385.

[16] Duarte E, Marco E, Muniesa JM, et al., Trunk control test as a functional predictor in stroke patients, J Rehabil Med. 34, 2002, 267272.

[17] Hsieh CL, Sheu CF, Hsueh IP, Wang CH, Trunk control as an early predictor of comprehensive activities of daily living function in stroke patients, Stroke. 33, 2002, 2626-2630.

[18] Susan S. Adler, Dominiek Beckers and Math Buck, PNF in practice, an illustrated guide (Third edition. Germany: Springer Publication; 2008).

[19] Peggy R Trueblood, Joan M Walker, Jacquelin Perry and JoAnne K Gronley, Pelvic exercise and gait in hemiplegia, PHYS THER. 69, 1989, 18-26.

[20] Ray-Yau Wang, Effect of proprioceptive neuromuscular facilitation on the gait of patients with hemiplegia of short and long duration, PHYS THER. 74,1994, 1108-1115.

[21] Glen and Gillen, Stroke Rehabilitation: A Function Based Approach (Third edition. United State of America: Elsevier Publication; 2011).

[22] Cynthia C. Norkin, D. Joyce White, Measurement of Joint Motion. A Guide to Goniometry (Third edition. Jaypee Brother Medical Publication Limited).

[23] American Medical Association: Guides to the Evaluation of Permanent Impairment (Third edition. American Medical Association: Chicago; 1988).

[24] Anne D. Kloos, Vanina Dal Bello-Haas, Rachel Thome et al., Interrater and Intrarater Reliability of the Tinetti Balance Test for Individuals with Amyotrophic Lateral Sclerosis, Journal of Neurological Physical Therapy 28, $2004,1$.

[25] Geert Verheyden, Luc Vereeck, and Steven Truijen, Additional Exercises Improve Trunk Performance After Stroke: A Pilot Randomized Controlled Trial, Neurorehabil Neural Repair 23, 2009, 281.

[26] WimSaeys, Luc Vereeck, Steven Truijen et al., A Randomized Controlled Trial study of Truncal Exercises Early After Stroke to Improve Balance and Mobility, Neurorehabil Neural Repair.15, August 2011.

[27] S. Karthikbabu1, Bhamini K. Rao, N. Manikandan, John M. Solomon, M. Chakrapani, and Akshatha Nayak, Role of Trunk Rehabilitation on Trunk Control, Balance and Gait in Patients with Chronic Stroke: A Pre-Post Design, Neuroscience \& Medicine2, 2011, 61-67.

[28] Deletis V., Dimitrijevic, M.R., and Sherwood, A.M., Effects of electrically induced afferent input from the human motor cortex, Neurosurgery20, 1987, 195-197.

[29] Benecke, R., Meyer, B.V., Gohmann, M., and Conrad, B., Analysis of muscle responses elicited by transcranial stimulation of the corticospinal system in man, Electroencephalography and Clinical Neurophysiology69, 1988, 412-422.

[30] Merton, P. A., and Morton, H. B., Stimulation of the cerebral in the intact human subject, Nature285, $1980,227$.

[31] Barker, A. T., Jalinious, R., and Freeston, I. L., Non-invasive magnetic stimulation of the human cortex, Lancet2, 1985, $1106-1107$.

[32] Kuniyoshi Shimura, and Tatsuya Kasai, Effects of Proprioceptive Neuromuscular Facilitation on the initiation of voluntary movement and motor evoked potential in upper limb muscles.Human Movement Science 21, 2002, 101-113.

[33] Knott M, and Voss DE, Proprioceptive Neuromuscular Facilitation: Patterns and Techniques (Second edition. New York: NY, Harper \& Row, Publishers Inc; 1968).

[34] Verheyden G, Nieuwboer A, Feys H, Thijs V, Vaes K and De Weerdt W, Discriminant ability of the Trunk Impairment Scale: a comparison between stroke patients and healthy individuals, Disabil Rehabil27, 2005, 1023-1028.

[35] Hindle, Tyler J. Whitcomb, Wyatt O. Briggs, and Junggi Hong, Proprioceptive Neuromuscular Facilitation (PNF): Its Mechanism and Effects on Range of Motion and Muscular Function, Journal of Human Kinetics 31, 2012, 105-113.

[36] Ruth Dickstein, ShragaHocherman, Thomas Pillar et al., Stroke Rehabilitation: Three Exercise Therapy Approaches. PHYS THER. $66,1986,1233-1238$.

[37] Rowlands AV, Marginson VF, and Leej, Chronic flexibility gains effect of isometric contraction duration during proprioceptive neuromuscular facilitation stretching techniques, Res Q Exerc Sport. 74(1), 2003, 47-51.

[38] Irwin OC, Proximodistal differentiation of limbs in young organisms, Psychol Rev 40, 1933, 467-477.

[39] McGraw M., Grasping in infants and the proximo-distal course of growth, Psychol Rev 40, 1933, $301-302$.

[40] Voss DE, Proprioceptive neuromuscular facilitation, Am J Phys Med.46, 1967, 838-898.

[41] Tyson SF, Trunk kinematics in hemiplegic gait and effect of walking aids, Clin Rehabil13, 1999, $295-300$.

[42] Messier S, Bourbonnais D, Desrosiers J and Roy Y, Dynamic analysis of trunk flexion after stroke, Arch Phys Med Rehabil85, 2004, 1619-1624.

[43] Michael Barnes, Bruce Dobkin and JulienBogousslavsky, Recovery after stroke (Cambridge university press; 2005). 
[44] Nudo R, Milliken G, Reorganization of movement representations in primary motor cortex following focal ischemic infarcts in adult squirrel monkeys, J Neurophys.75, 1996, 2144-2149.

[45] Nudo R, Wise B, Sifuentes F, and Milliken N, Neural substrates for the effect of rehabilitative training on motor recovery after ischemic infarct, Science.272, 1996, 1791-1794.

[46] Classen J, Liepert J, Wise SP, Hallett M, and Cohen LG, Rapid plasticity of human cortical movement representation induced by practice, J Neurophysiol.79(2), 1998, 1117-1123.

[47] Karni A, Meyer G, Jezzard P, Adams MM, Turner R, and Ungerleider LG, Functional MRI evidence for adult motor cortex plasticity during motor skill learning, Nature. 377(6545), 1995, 155-158.

[48] Nestor A. Bayona, Jamie Bitensky, Katherine Salter, and Robert Teasell, The Role of Task-Specific Training in Rehabilitation Therapies, Stroke Rehabil. 12(3), 2005, 58-65. 